\title{
Mobile Information Retrieval in a Hybrid Peer-to-Peer Environment
}

\author{
Kam Fung Yeung, Yanyan Yang \\ Dept. of Electronic and Computer Engineering, University of Portsmouth \\ Anglesea Building, Anglesea Road, \\ Portsmouth, Hampshire, PO1 3DJ, United Kingdom \\ +442392 842543
}

\begin{abstract}
The proliferation of wireless and mobile devices has created a large demand for mobile information content as well as effective mobile information retrieval. In this paper, we present JHPeer, a software framework for mobile information retrieval in hybrid peer to peer (P2P) networks. JHPeer incorporates context engine to fit on mobile devices to enable context-based personal information retrieval. A prototype system has been developed based on JHPeer framework for retrieving, indexing and sharing user information on mobile devices in P2P environment.
\end{abstract}

\section{Categories and Subject Descriptors}

C.2.4 [Computer-Communication Networks]: Distributed Systems - distributed applications; H.4.3 [Information System Applications]: Communications Applications - information browsers; H.5.3 [Information Interfaces and Presentation]: Group and Organization Interfaces - collaborative computing

\section{General Terms}

Management, Documentation, Performance, Design, Experimentation, Human Factors, Standardization.

\section{Keywords}

Peer-to-Peer, Hybrid Framework, JXTA, Context-awareness, Information Retrieval

\section{INTRODUCTION}

Traditional information retrieval techniques were developed to achieve efficient document retrieval over a collection of documents. These later on, started to focus towards more hybrid and distributed information systems such as the World Wide Web. Contemporary web search engines and web directories target desktop users by returning them documents based on user queries. Similar with accessing to an existing www contents isn't just enough for a mobile user. With the realization of pervasive computing technologies, user surrounding is becoming more and more information rich. In such an environment, a typical mobile user will come across different information sources while being on the move. The nomadic nature of user itself puts a lot of

\footnotetext{
Permission to make digital or hard copies of all or part of this work for personal or classroom use is granted without fee provided that copies are not made or distributed for profit or commercial advantage and that copies bear this notice and the full citation on the first page. To copy otherwise, or republish, to post on servers or to redistribute to lists, requires prior specific permission and/or a fee.

Mobility 2009, Sep 2-4, Nice, France Copyright (C) 2009

ACM 978-1-60558-536-9/00/0009.....\$5.00
}

constraints and brings about a lot of dynamics to user's context. Mobile users' information needs change as they change their location as well as other contextual attributes such as task, emotional or environmental context. The centralized system cannot satisfy the mobile user's demand for effective information retrieval and sharing due to the rapid growing of mobile users seeking for dynamic information. Being on the move, the organization of communication network will be highly dynamic for both the user and the information.

As the advent of wireless technologies and constant development of mobile devices, the ability of sharing information through a P2P service on mobile devices has become a promising alternative to centralized approach for large-scale data retrieval and sharing. A range of wireless network technologies such as Bluetooth, Wi-Fi (Wireless Fidelity), 3G cellular network or WiMAX (Worldwide Interoperability for Microwave Access) that fitted on ubiquitous mobile devices are common. These mobile networks allow mobile users to access for information anytime, anywhere and provide users with satisfactory average bandwidth for P2P communication rather just browsing web or email reading. Additionally, mobile network operators also have acknowledged the ubiquitous mobile services that are the obvious trend. They are offering their customers unlimited Internet access with a lowered price [2]. This leads to a growing importance of information services on the move. However the quality and quantity of in-time personal information services available for mobile users still lag behind.

In this paper we present a software framework, called JHPeer (JXTA [1] based Hybrid Peer to Peer), for context based mobile information retrieval and sharing in hybrid peer to peer (P2P) networks. JHPeer is a generic framework which enables the development of software applications for mobile environment such as Mobile Map, Mobile Blog, Tourism, Social Collaboration and personalized mobile information retrieval. This paper shows how the functionality required for personal mobile information sharing applications can be achieved through a set of application components in a simple way, and how to provide in-time contextsensitive information services to mobile users in a in P2P fashion. A prototype system has been built based on the proposed framework.

The rest of this paper is organized as follows. In section 2, we briefly review related work. Section 3 describes JXTA based Hybrid Peer to Peer (JHPeer) framework and introduce the main considerations of the design. Section 4 explains how we model the context. Section 5 discusses the application scenarios. In section 6, we report on the implementation status of our system. We conclude in section 7 . 


\section{RELATED WORK}

Several frameworks $[3,4,7,8]$ have been previously proposed to implement context-aware application. CASS [3] is a server-based middleware that facilitates context-aware application on mobile devices. Because of the server-based approach, it suffers from central server failure and memory/processor constraints. SOCAM [4] is ontology-based context-aware service focused on a smart space domain. It uses OWL to resolve the issues of context modeling and context reasoning. However, it is implemented in Java RMI technology and is not suitable for large-scale network. CoBrA [7] is an agent-based framework that deploys intelligent context broker to receive, integrate and share the contexts. CMF [8] is designed for context management for processing and exchanging context information in mobile environment. It aims to operate in pure peer-to-peer framework.

Previous approaches adopt semantic technology for developing context-aware application. However, most of them either rely on centralized service or operate on a pure peer to peer fashion. The former approach suffers from a single point of failure and has limited scalability while the latter approach may overload the network and is less efficient. JHPeer uses hybrid approach with multiple super peers to address these problems. JHPeer incorporates information retrieval engine which uses contextawareness for effective information retrieval in such environment.

\section{THE JHPEER FRAMEWORK}

Figure 1 presents the hybrid P2P framework of JHPeer.

JHPeer framework is designed to enable the development of software applications for mobile environments. The framework follows most of desktop based peer to peer systems in that each node is equal and performs both client and server functionality. It consists of 2 types of Peers - Super Peer and Mobile Peer. The existence of the super peer creates a slightly different framework from the standard P2P framework in which no central server exists.

Both Super Peer and Mobile Peer incorporate an information processing engine which supports the filtering of content with

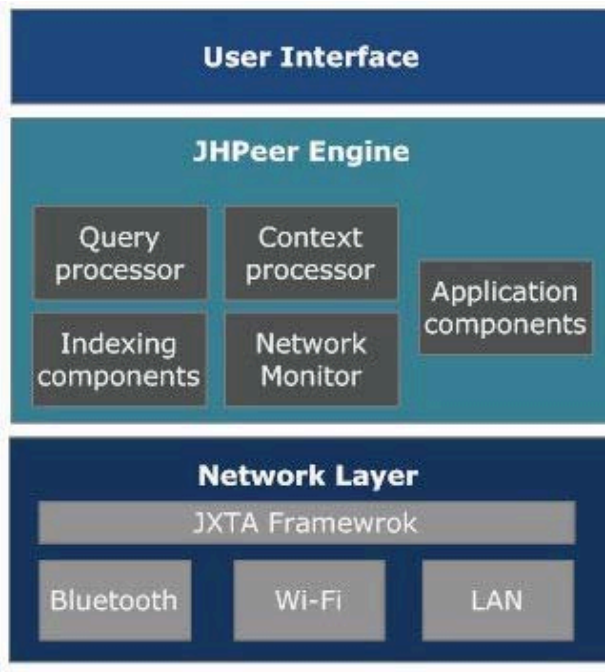

Super Peer respecting to user preference and context information, providing effective information retrieval in a $\mathrm{p} 2 \mathrm{p}$ fashion. The difference between super peers and mobile peers are that super peers are responsible for creating and monitoring the overlay network and providing searching and directory services to other super peers and mobile peers. Super peers also provide message propagation and knowledge caching. Mobile peers dynamically join to the networked community and provide information for sharing, and allow context based retrieval. The functions of each component are described as follow:

Query Processor - handle and process queries, allow context based retrieval.

Context Processor -manage and share context information in the Peer Group network.

Indexing Components - organize information into index file for fast searching and save temporary information in the database.

Network Monitor - monitor the peer network and check for online peers.

Application components - are services components deployed in the system for providing functionality to users.

\section{CONTEXT MODELING}

A Context is the information about a person's proximate environment. Pervasive System will use the context information on decision and anticipating user needs. Proactive information services which automatically retrieve relevant information for user would help to meet user expectation transparently. Context information can be divided into static and dynamic context. Static Context refers to context that is seldom changed during service usage such as personal calendar/schedule, address book, contact list, to-do list, user profile and hardware profile. Dynamic Context is the frequency changes information and usually is the information that is gathered by sensors like location, temperature, time, speed and emotion.

In order to enable context-aware computing, contextual information needs to be modeled and allows application to interpret relevant context. Key-value model is used to tag context

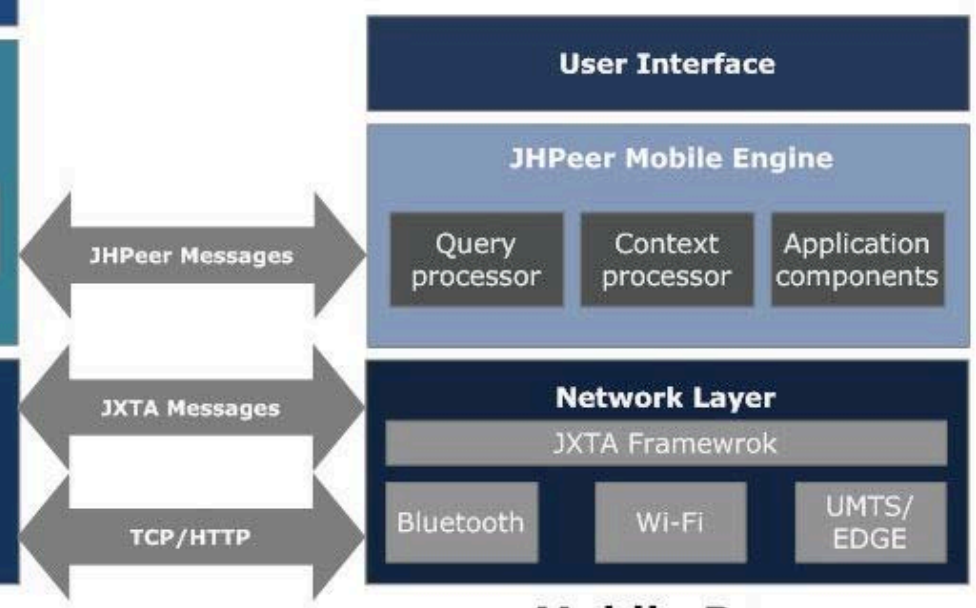

Figure 1. JHPeer Framework 
data inside a XML message for the purpose of distributing context resource among Peers. Each piece of contextual information is tagged inside the Tag < jhpeer:Context $>\ldots<$ jhpeer:Context $>$ in the XML Message. For increasing the flexibly of our framework, the content of context information could be constructed from structured XML Schema, Resource Description Language (RDF) [5] or Web Ontology Language [6] depending on the requirement of the service.

Examples for location-aware services such as various social services allow locating your friend on the maps and finding the distance. These gather position information from various sensors includes GPS, AGPS, WiFi, RFID and mobile phone tower (CellID). This type of services will require Location-ontology based XML for communication. Several models could be used such as RDF, KML [9] or custom XML Schema. For RDF, <geo:lat> and <geo:long> could represent the global position. For $\mathrm{KML}$, it is designed for expressing geographic annotation which contains additional information like place mark description, icon, style...etc. For custom XML, context attributes need to be defined. A couple of attributes include name, position, address are required. Additionally, attributes like timestamp, gathered sources, accuracy will benefit the computation.

\section{JHPEER APPLICATIONS}

Several potential applications may be integrated into JHPeer System as follows.

\subsection{Tourism}

Tourism service could enhance tourist experiences, even locals. Once a user starts this application, system would provide local information depending on the geographical position. For example, a mobile Wi-Fi user accesses Portsmouth. The application would acknowledge the location where the user is via GPS/AGPS and offers some useful recommendation, e.g. address of the nearest Holiday Inn, special event of the popular pub and the sale information in nearby shopping centre. If the user selects the address of the nearest Holiday Inn, the system will provide further personal information, like the directions to Holiday Inn and price of the hotel for different service.

\subsection{Mobile News}

News Service could benefit mobile user by automatic publishing up-to-date relevant news and alerting user with interested topics using user's preference and contexts. Imagining that you arrive a city, the system will report the current local event, traffic information and weather situation. All these resource will show to the user without formulating any query. It will just be achieved by locating the user position. The most benefit of this service is that the user could make the decision timely by updating the news at the first time when it occurs.

\subsection{Mobile Discussion Board}

Discussion board could be applied in several areas, e.g. communicating the quality of one product or the situation of ongoing football game. Mobile discussion broad provides an information sharing platform to fulfill the demands of user to the fullest extent. The other good example is about the stock discussion platform. Because of the attribute of the stock market, it will produce plenty of data, and the stockholders need to exchange the information as fast as possible. Offering a mobile discussion broad is desirable for the specific market environment.

\subsection{Mobile Blog}

Nowadays, creating users' own blog and expressing themselves about anything have become the habit of people. It is the approach that allows people to record the current feeling. However, since the limitation of desktop blog, blog likes an online dairy than an emotion note. Mobile blog lets user to share the moment which is happened. Also, in our system, people could read the mobile blog which is posted by the user who is in the same location. It will facilitate the communication between those mobile users the people next to you and share the feeling for the same event.

\section{STATUS OF IMPLEMENTATION}

We have prototyped JHPeer using Java and deployed it on a small network of mobile devices. Figure 2 shows a simple prototype that implement on HTC Touch emulators (Windows Mobile). It allows mobile user to search nearby resources and friends locations via published context information, which pushes interesting information to users based on user's context information rather than to require the users to search the information they need. Map/Friend Service enables user to find his friends nearby. Friend could be added and located by searching through the contact list in the mobile phone or manual search by user through email.

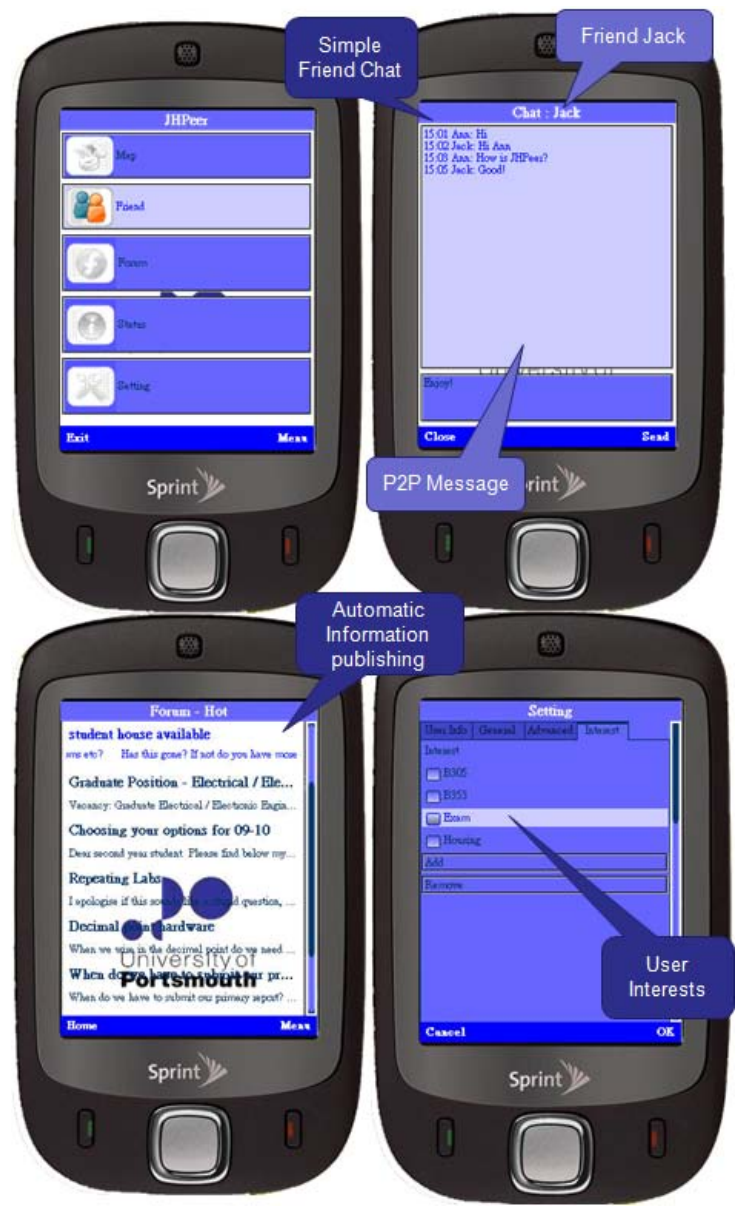

Figure 2. Prototype Screen Captures 
Different mobile users may be interested in different information services. We use departmental forum as an example service. As a scenario, Lee is a third year student. He is interested in all posts in his current units: B305, B353. He is also interested in the topics of housing and exam since the exam is coming and he needs to move out from the hall of resident soon. With the support of Super Peer that will automatically push the most relevant topics to his mobile devices that he may be interested in. So he won't miss any important posts. He is able to add or remove any interest in the user profile through the Setting Interface.

We carried out some initial experiments in order to evaluate the performance of the developed prototype. We publish around 15,000 advertisements to the Peer Group in our test. These advertisements include user preferences, user contexts and the content of forum discussion. The average time taken for results to be received after a query was broadcasted was 800 milliseconds (ms). The value is acceptable for the prototype system to be used in future user experiments. Table 1 shows the detail of cost for processing a forum request. The mobile device took about $2 \mathrm{~ms}$ to generate the query XML message and passed it to JXTA Pipe Service Queue for transmission. It took about $50 \mathrm{~ms}$ to pass to other peer through Local Network, while the time is depended on network condition and sizes. The Forum Service in Super Peer only needed around $20 \mathrm{~ms}$ to process and response the query. However, the result was usually a list of Thread data that required more time to send back to mobile peer. Mobile peer would handle the message and parser the XML to runtime variable. This process is various due to the size of message.

Table 1. Transmission Cost

\begin{tabular}{|l|l|}
\hline Task & Simulated (ms) \\
\hline Mobile Peer Message formulation & 2 \\
\hline Message in-Queue & 10 \\
\hline Transmission to Super Peer & $50 \sim 100$ \\
\hline Super Peer Service Process Time & $\sim 20$ \\
\hline Transmission to Mobile Peer & $100 \sim 500$ \\
\hline Mobile Peer JXTA Message handle time & $100 \sim 400$ \\
\hline Mobile Service Message parser time & $2 \sim 14$ \\
\hline
\end{tabular}

\section{CONCLUSION}

In this paper, we describe a JXTA based Hybrid Peer to Peer context-aware system for mobile information service. The JHPeer system allows distributed access, concurrent querying and parallel computing for mobile information retrieval system over a large scale of mobile network. JHPeer deploys a Query and Context Processor to process context information and provides in time and personal information for mobile users. The JHPeer system can grow while adding new super peers and mobile peers.
Previous research has been focused on pure P2P technologies or relied on a single centralized directory service. What we are doing is to build the foundation of peer to peer technology and JXTA platform for context based searching and sharing of mobile data, and put this work into engineering practices. Our investigation is just at the beginning, and far from accomplishment. However, we believe that autonomous and scalable environment is necessary for efficient information sharing in a mobile context. In the near future, we will try to build such an environment for mobile users and investigate the cost, performance and energy consumption of this system.

We are currently working on implementation of context based information retrieval, a better messaging delivery mechanism over JXTA network to overcome potential disconnection of mobile wireless devices, and implementation of network connection APIs over Wi-Fi, Bluetooth and cellular network access point.

\section{REFERENCES}

[1] JXTA. 2009. JXTA Community Projects. https://jxta.dev.java.net/.

[2] Richardson, T. 2006. T-Mobile to make mobile net service 'unlimited'. The Register. http://www.theregister.co.uk/2006/03/24/t_mobile_unlimited.

[3] Fahy, P. and Clarke, S. 2004. CASS - a middleware for mobile context-aware applications. In Workshop on Context Awareness, MobiSys 2004.

[4] GU, T., Pung, H. K. and Zhang, D. Q. 2005. A serviceoriented middleware for building context-aware services. Journal of Network and Computer Applications, Vol 28 issue 1, 2005, pp. $1-18$.

[5] Herman, I. Swick, R. and Brickley, D., 2004. Resource Description Framework (RDF)/W3C Semantic Web Activity. http://www.w3.org/RDF/

[6] Herman, I. 2004. Web Ontology Language OWL/W3C Semantic Web Activity. http://www.w3.org/2004/OWL/.

[7] Chen, H., Finin, T. and Joshi, A. 2003. An ontology for context-aware pervasive computing environments. The Knowledge Engineering Review (2003), 18, Cambridge University Press, pp. 197-207.

[8] Van Kranenburg, H., Bargh, M. S., lacob, S. and Peddemors, A. 2006. A Context Management Framework for Supporting Context-Aware Distributed Applications. IEEE Communications Magazine, vol 44, issue 8, pp 67-74.

[9] KML. 2009 Keyhole Markup Language. http://www.opengeospatial.org/standards/kml/ 